\section{Conflicto existencial en La agonía del poeta de Rolando Steiner}

\author{
Existential conflict in the agony of the poet of \\ Rolando Steiner
}

Mayra Josefa Bonilla Martínez Universidad Nacional Autónoma de Nicaragua, Managua mayraartista@gmail.com

(C) UNAN-Managua

Recibido: abril 2018 Aprobado: junio 2018

\section{RESUMEN}

Steiner muestra en su quehacer teatral y específicamente en esta obra que analizamos una preocupación por el trabajo creador del artista y una soledad interior permanente. Interpretamos el conflicto existencial del personaje protagonista (Félix) teniendo en cuenta que los existencialistas plantearon problemas relacionados con el sentido de la vida, la elección, responsabilidad personal y el destino del hombre y que el fenómeno ontológico existencial es producto de esos conflictos internos del entorno y los seres que acompañaron al poeta a través de su vida. La visión del mundo plasmada en la obra La Agonía del Poeta, corresponde a la visión del autor que desde el punto de vista religioso presenta a un Félix, temeroso ante lo inesperado, la angustia de vivir, la incertidumbre a lo desconocido y lo que hay después de la muerte. El protagonista experimenta esos dos momentos vitales del ser: la permanencia y la muerte. El primero que significa perpetuarse a través del ideal y pasión que para el fue su proyecto de vida, la poesía y el segundo la muerte, como un hecho ineludible de angustiosa realidad en la cual no tiene elección ni poder para decidir sobre su destino.

Palabras clave: : existencialismo, teatro, críti$\mathrm{ca}$, Rolando Steiner

\section{SUMMARY}

Steiner shows in his theatrical work and specifically in this work that we analyze a concern for the creative work of the artist and a permanent interior loneliness. We interpret the existential conflict of the main character (Felix) considering that the existentialists raised problems related to the meaning of life, choice, personal responsibility and the destiny of man and that the existential ontological phenomenon is the product of those internal conflicts of the environment and the beings that accompanied the poet throughout his life. The vision of the world embodied in the work The Agony of the Poet, corresponds to the vision of the author who from the religious point of view presents a Felix, fearful of the unexpected, the anguish of living, the uncertainty of the unknown and what there is after death. The protagonist experiences these two vital moments of being: permanence and death. The first one means to perpetuate oneself through the ideal and passion that for him was his life project, poetry and the second death, as an inescapable fact of anguishing reality in which he has no choice or power to decide on his destiny.

Keywords: existentialism, theater, criticism, Rolando Steiner 
El teatro de Rolando Steiner (1936-1987) es de gran importancia dentro de la literatura nicaragüense. Se considera uno de los más representativos del teatro moderno contemporáneo que se produce a finales de la década de los años cincuenta, y logra sus mejores momentos en las décadas del sesenta y setenta. Abordó temas de la incomunicación, soledad, muerte y la angustia existencial. De ahí el interés de estudiar su teatro. En este trabajo interpretaremos el conflicto existencial del personaje (protagonista) en la obra La agonía del poeta teniendo en cuenta que los existencialistas plantearon problemas relacionados con el sentido de la vida, la elección, responsabilidad personal y el destino del hombre. Es decir la vivencia de la existencia.

Steiner muestra en su quehacer teatral una preocupación por el trabajo creador del artista y una soledad interior permanente. El fenómeno ontológico existencial fue producto de sus conflictos internos y de las circunstancias que le rodearon. En esta obra se advierte un intento - dice Valessi (1991) - de establecer un paralelo entre la angustia que vivió el genial poeta en la antesala de lo inexplicable y la propia angustia del autor... "Capté que Steiner, al escribir la obra, no tuvo preocupación por desarrollar una intriga ni un carácter, descubrí entre líneas la existencia de símbolos provocativos sembrados no con intención catártica, sino para resolver agresivamente complejos viscerales del espectador, angustias metafísicas de sello universal" (p. 2). La obra de Rolando Steiner es moderna, actual y de mucho valor para la literatura dramática nicaragüense que ha estado mas preocupada por un realismo referencial que por el sentido ontológico.

En la Teoría y técnica de la dramaturgia, John Howard Lawson (1976) expresa: "el carácter esencial del drama es el conflicto social. Este conflicto tiene que ver con la conducta del hombre en relación con otros hombres o con su medio" (p. 271). El conflicto dramático propone el ejercicio de la voluntad consciente y debe ser riguroso como para sostener y desarrollar el conflicto hasta llegar a su desenlace. Por su parte Bernard Shaw, (citado por Villegas, 1982) afirmó: "Todos los dramas deben presentar un conflicto. El desenlace puede ser reconciliación o destrucción, o como en la vida misma puede no haber tal desenlace. Pero el conflicto es indispensable, sin conflicto no hay drama" (p. 37) sin embargo, en estos criterios de análisis, se observa también que el conflicto en La agonía del poeta, se da en el primer acto, donde se hace la presentación el desarrollo hasta llegar al desenlace.

Félix - (Irritado) ¡Deja de protestar a todo lo que digo!... No temo a la muerte... En ocasiones he gozado como no lo han logrado los millonarios de esta tierra; he comido y vestido como príncipe, y como ellos, me he relacionado con las personalidades del mundo. He derrochado dinero, que gané en abundancia. Y con frecuencia he sentido el aletazo de la gloria... ¿Qué me queda por desear?... ¿Decrepitud? ¿Idiotez senil? ¡No! Que venga la muerte.

La postura ante la muerte, de Félix, es auténtica, en la que su estado anímico de la ansiedad o angustia ha recuperado el ser para la muerte, como un modo de permanencia y recuperación del ser total. Félix, acepta la realidad y la libre elección de su destino. El conflicto fundamental surge de la agonía de Félix y sus relaciones con los otros seres, ante la fatalidad de la muerte. La acción dramática se realiza entre el inconsciente y la realidad, suceden episodios entre el sueño y la vigilia en el plano en que aparecen los seres que han muerto (la madre, su esposa "Stella", muerte).

Estas situaciones antes mencionadas parecen no tener relación con el conflicto principal, pero constituyen el sentido central de la acción dramática, la muerte de Félix. Steiner recurre una vez más al surrealismo (ver Judit) en donde la vigilia pierde prestigio frente al sueño y el subconsciente. 
La construcción dramática en ese momento se fundamenta en el motivo de la búsqueda de afecto, (de amor y ternura). El protagonista busca este amor de la madre y de la esposa ausente, anhela estar cerca de ellas aunque sea en el mundo de los sueños. En su mundo real está Emelina.

\section{a- Conflicto interno del personaje}

La acción dramática se sustenta en el acoso progresivo de la muerte al protagonista Félix, a causa de su enfermedad. La obra se estructura en tres actos los cuales constan de escenas y cuadros. El avance de la acción dramática está impulsado por los conflictos internos del personaje, tal como lo describimos a continuación:

Sec. 1 Presentación inicial del ambiente y las pesadillas que manifiesta el protagonista (Félix)

1.1 Discusión entre Félix y Emelina (esposa), reproches y reclamos.

Sec. 2 Reflexión de Félix, por tanto odio acumulado de Emelina.

2.1 Entrada de la mujer (su madre), recuerdos de su madre y así exclama:

Félix-... ¡ Una madre muerta nunca es un espectro y sin embargo, evocarla me llena de zozobra! (El anhelo de búsqueda de sí mismo le llena de desasosiego. La incapacidad del profundizar en un recuerdo consolador, era una sensación de soledad y vacío).

Sec. 3 Llegada de Emelina a los gritos de Félix, llamando a la mujer.

3.1 Emelina tranquiliza a Félix y recuerdan cuando se conocieron.

3.2 Nuevamente se reprochan (Félix - Emelina)

Emelina - ¡Y que poco amor guardabas, que no quisiste perdonarme!... ;Me sacrificaste por amor propio, burlándote de mis sentimientos!... iTe fuiste del país, y te casaste con Stella, como si yo no existiera!

Félix-(reclamando)... ¡Nunca tuviste amor propio!

De nuevo el enfrentamiento de Félix con Emelina (su esposa) y cada uno justifica las razones y motivos de su comportamiento: Huída, búsqueda de refugio y búsqueda de sí mismo en el otro.

En todo el primer acto, se da el enfrentamiento del protagonista (Félix) con el antagonista (Emelina), quien reprocha y recrimina a Félix por sus actos. El otro enfrentamiento de Félix es con los fantasmas o espectros que le aparecen, la presencia de estas visiones se confunde con lo agónico real y su propia existencia. El temor de Félix hacia la muerte, al más allá, a lo desconocido a la nada, es por tener conciencia de la experiencia reveladora de la vida y la muerte como una autenticidad del existir. El núcleo de la acción se centra en torno al acosamiento real (Emelina) y el imaginario (espectros- madre-Stella), que lo llevan a Félix a ese estado de temor, angustia, incertidumbre y soledad, en su deseo por romper o unir ese vínculo que les une. 
Para los existencialistas, la "autenticidad" supone un verdadero proceso de apropiación de culpa del hombre que implica el arrepentimiento y el nacimiento del hombre nuevo. A propósito de este tópico, la angustia, dice Sartre, "es la total ausencia de justificación así como la plena responsabilidad respecto de todo y de todos" (Arrien, 1978:201). Cada uno de los personajes, buscan y encuentran el motivo de su accionar, los exponen, presentan razones de su proceder de acuerdo a las circunstancias, época y necesidad de adquirir un compromiso, una responsabilidad. Así lo expresa Félix y la mujer (madre).

Mujer - Pero... ¿Qué mal le había provocado ella, para humillarla así? ¿No le importó el daño irremediable que le causaba?

Félix - (silencio) ¡No!... Quería que sufriera el horror de sentirse repudiada, como yo lo habia sufrido. Que sintiera en su carne mi dolor.

Mujer - (con profunda ternura) iPobre hijo!

En este diálogo los dos personajes explican la razón que les impulsó a actuar de esa forma y expresar sus verdaderos sentimientos. "Es necesario volver atrás, al pasado, para hacerlo nuestro en un acto de apropiación existencial, en donde el hombre acaba por reconocer su culpa o más bien su ser culpable... Es doloroso pensar en la existencia porque es difícil eludir la responsabilidad moral que supone el vivir en este mundo y el estar con los demás" (Robert, 1973, p.115). Se debe sentir y experimentar la problemática del ser humano para reconocer su propia posibilidad de ser.

\section{b- Dualidad de las acciones: Acción interna y externa}

El drama La agonía del poeta está construido sobre la base del desarrollo de dos acciones simultáneas, cuya interrelación origina el movimiento de ambas. El avance de la acción externa (discusión y reproches de Emelina) conduce hacia la iluminación retrospectiva de la interna (analepsis). El dramatismo del texto proviene del enigma que se va descubriendo progresivamente en torno al acoso de Félix y del vigor de las pasiones que participan, vivencias del poeta, su gran pasión hacia la creación "La poesía". La acción interna (analepsis) corresponde a los amores de Félix (Rubén) y las circunstancias trágicas que no hicieron posible su realización, el amor de su madre, el amor de su Stella. (Experimenta la terrible y dolorosa soledad del hombre - artista).

La acción externa corresponde a la relación de Félix con su esposa Emelina, la cual es forzada por conveniencia y despecho. A medida que avanza la acción se van descubriendo los verdaderos sentimientos de Emelina: Amor, odio, celos, despecho, venganza. Emelina (Rosario Murillo) en su empeño de establecer y mantener una relación con Félix, lo lleva a su propio aniquilamiento y a la destrucción del mundo de ambos.

Desde el punto de vista organizativo, en la obra funcionan paralelísticamente las dos acciones de forma continua y con un vínculo íntimo entre una y otra. La primera corresponde al presente de Félix, que se encuentra enfermo, agobiado por el dolor interno, el recuento de sus actos y los dolores ocasionados por la enfermedad. El obstáculo a su voluntad proviene de su relación familiar con Emelina, quien reprocha cada una de las actitudes que el esposo ha tenido al abandonarla. El conflicto da origen a una acción interna (la segunda) que se va exteriorizando retrospectivamente durante el proceso en que se desarrolla la acción real. Esta acción se presenta en gran parte del drama como una fuerza que impulsa y motiva a la otra. Se manifiesta a través de análisis de sus actos (delirios ocasionados por la fiebre o el dolor físico). 
La acción principal del drama, como un todo organizado es la externa, (reproches y reclamos de Emelina). El no tener una relación estable con Félix (convencionalismos) provoca inconformidad y despecho. El inicio de este desamor, desconfianza e inestabilidad por parte de Félix, tiene sus raíces en su infancia, cuando él apenas, si conoció a su madre. Esta búsqueda del amor maternal como un refugio a la soledad experimentada cuando era niño, le lleva y conduce a otras revelaciones, las cuales se manifiestan tanto en la acción interna como en la externa. En el primer delirio aparece la sombra borrosa de la mujer (Rosa Sarmiento), su madre; en el segundo, la imagen de su primera esposa (Rafaela Contreras), su Stella; y en el tercero, la muerte. En la realidad, Emelina, su segunda esposa, cruel y real es como una sombra que acecha cada momento del moribundo. Le cuida y hostiga con reclamos y recuerdos, haciéndole menos dulce sus últimos momentos de existencia.

En el primer acto aparece el conflicto externo. El tema como su nombre lo indica es la agonía, no de un hombre indeterminado, sino la del poeta como símbolo y representación de un país. Félix experimenta el acoso de parte de la mujer a quien él más ha repudiado como esposa (Emelina). La existencia del protagonista Félix es asediada también por los seres que en su vida han constituido ese vínculo familiar (otra vez este vínculo en la obra de Steiner) desde la madre hasta la esposa que le vio morir.

El personaje Félix tiene carácter protagónico, se describe como un ser pesimista, temeroso, lleno de ansiedad e incertidumbre, sin esperanzas. Se observa en el primer diálogo (delirio) con la mujer (su madre) y en los reproches de Félix, su abandono y traición. Ella justifica y cuenta los motivos de su conducta, da las explicaciones del por qué se alejó. Termina este primer acto con los reclamos de Emelina hacia Félix, por el abandono y lo hace recordar algún momento agradable en su relación.

La organización de los hechos revela el predominio de la acción externa y la ausencia de referencias claras y bien definidas de la acción interna a través de una analepsis que se da en el primer acto. El personaje central, comienza a ser definido y es cuando el lector conoce las razones de su actitud. Al inicio de la acción (externa) se experimenta el planteamiento del estado de gravedad en que se encuentra Félix y la razón de los delirios que sufre. En la segunda fase se nos presentan claramente definidos los conflictos internos en la relación de la pareja (Emelina - Félix). En el primer delirio la acción (interna) se da a partir de una analepsis y es la llegada de la mujer con la que Félix conversa y habla de su madre.

Félix... Crecí vacío de ternura... ¡Huraña, silenciosa, solitaria! Igual a esos arbustos, secos $y$ desolados que crecen al borde de los caminos...

En este encuentro se plantean claramente la desconfianza, el abandono, la soledad, el adulterio, la fuga y el divorcio.

Félix expresa: Jamás le importó que añorara su cariño... iQue vagara en mi alma como una lacerante angustia...! ;Me abandonó y se fue a vivir lejos de mí, libre de ataduras conyugales!...

Esta es una escena que nos permite conocer los antecedentes de Félix, su relación filial tan determinante en esa temprana edad y que le marcará durante toda su vida. Es la cotidianeidad del individuo que se da entre el nacimiento y la muerte, el diálogo es emotivo y angustioso; Félix revela el desamor, resentimiento y sobre todo el dolor que le causó la ausencia de su madre. 
La sección siguiente nos lleva nuevamente a la acción externa, la realidad. Félix y Emelina discuten, pero luego se dejan llevar por los recuerdos de juventud, al instante surgen los reproches y recriminaciones de ambos personajes. Esta escena es importante, en función de la historia porque se conocen los detalles de esa relación forzada, por obligación y compromiso, que si en un momento hubo amor, este fue perdiendo la ilusión y las posibilidades que la pareja tenía de ser feliz, así lo observamos en el siguiente parlamento:

Félix - iMe exilaste, brutalmente, del amor y de la felicidad!

Emelina - ¡Ambos hemos sido desdichados!

Concluye esta escena con las explicaciones individuales del por qué de su proceder; cada uno ha actuado de acuerdo a su amor propio, de una manera egoísta y personal. Se percibe una profunda desesperación y soledad, que pone de manifiesto el fracaso en la relación. Fracasan la amistad, el amor en su única aspiración de un vínculo de posesión, de permanencia por parte de Emelina.

El acto segundo se inicia con la acción externa, la presencia del Dr. Dubois, quien examina a Félix y da su diagnóstico, el cual sugiere una operación, a lo que Félix se niega rotundamente. En este acto el protagonista (Félix) tiene un monólogo en el que se interroga sobre la existencia de Dios, la muerte, la vida y el dolor de estar vivo.

Félix -... ¿pero vale tanto morir, para sufrir tanto?... ¿Y si después del más allá, sólo existe el abismo, la nada?... ¡Dios mío!... ¡Tienes que existir!... ¡Sería una terrible burla que no fueras...

Este tipo de situaciones de la quietud y la muerte, supone para Jaspers un proceso continuo de lucha, y se encuentra ligada a la angustia de carácter fáctico. El coraje para enfrentarse a la angustia mortal...Se trata de un coraje para morir verdaderamente sin ningún auto engaño... (Ibid, 207) Félix, se enfrenta a la realidad de la muerte como algo inesperado, imprevisto, y continúa... iy si muero!... ¿Qué seré, qué será de mí, entonces?... ¿y qué es la muerte? ¿No respirar?... ¿no sentir?... ¿no habitar sobre la tierra? ¿No ser medido por el tiempo?... ¿Es el no absoluto, entonces?... ¡La muerte existe, y no es dormir!...La muerte, para Sartre, es un hecho exterior que viene de fuera y por tanto absurdo y contingente como el nacimiento. "La muerte es un hecho contingente que se refiere a la facticidad de la realidad humana, la finitud es una estructura ontológica del < para sí> que determina la libertad. Yo no soy libre para morir, sino soy un mortal libre" (Ibid, 210). Félix no quiere dormir, soñar, pensar ni sentir que es el castigo del hombre. Sin embargo no lo puede evitar y pasa de este estado de angustia y vacío al delirio (acción interna) otra analepsis, donde aparece su Stella, que inspira paz y alegría al alma atormentada del moribundo, es el reencuentro con la amada:

Félix - ¡Oh ángel, sueño, deseo mío!... ¡Cómo he anhelado verte! ¡Cómo he necesitado tu ternura, tu consuelo, tu fortaleza!

Al inicio y final del segundo acto, Félix, tiene dos monólogos intensos, que muestran en el mundo onírico: 1- El deseo de permanecer al lado de Stella y es la muerte quien interfiere en esa unión. 2- Félix, busca la salvación y establece un modo de comunicación con Dios, se aferra a la vida y explora todos los infortunios y placeres que ha disfrutado de ella. Aferrándose a la poesía dice: - Ella ha sido mi verdadera madre, esposa, mi hija, ¡Ella es lo que más amo, lo que más venero, lo que habla más íntimamente a mi corazón!... Termina este segundo acto donde se produce el encuentro con la muerte a quien Félix ve y describe como una siniestra y deforme sombra, pero no la nombra. 
Es una escena patética y de angustia para el protagonista que lucha con la muerte, creando por el contenido de sus palabras y su interpretación, una peripecia de la acción interna, que se reflejará más tarde en la acción externa.

Félix -... ¡He reconocido su semblante; pero he olvidado su nombre! ¡Anda como alimaña! ¡Arde su mirada como satanesa del Dante!... ¡Horrible bestia!... ¡Al irse me miraba siempre!... ¡Que no regrese jamás!...

Se habla de la muerte, como algo indeterminado, deforme, que amenaza con la mirada y está en guardia pendiente de los movimientos de su presa. A propósito de este tipo de fenómenos Heidegger, señala: "El morir desciende al nivel de un acaecimiento que alcanza sin duda al "ser - ahí", pero que no pertenece propiamente a nadie" (Ibid, 243). El protagonista huye de la angustia ante la incertidumbre del propio destino, dentro de la certidumbre del acaecimiento fatal de la existencia y despierta, regresando a la realidad.

El tercer acto está integrado por 3 escenas o situaciones dramáticas. En la primera y segunda escena queda resuelto y aprobado que Félix será sometido a la operación, una simple paracentesis según dice su médico, en la tercera escena o situación Félix, reflexiona emocionado al escuchar el canto gregoriano y cómo enfrentar este momento, sus creencias, su fe y los conflictos de sus ideas, su pensamiento es el del autor Steiner, así dice:

Félix - ¡Siempre temí enfrentarme a esta hora de mi vida, y he aquí que llega!... ¡Cada minuto ha sido consumado!... ;Todo es ya irrevocable!...

Este monólogo es una secuencia del monólogo final, únicamente cortado por las voces (canto gregoriano) que se escuchan fuera de la habitación. Termina este acto con el reconocimiento del protagonista, con el recuerdo de sueños y de seres que han estado a su alrededor y que ha pretendido expresar con su ser, su existir, su estar en la cotidianeidad de este mundo, aceptando a la muerte, seguro de permanecer vivo en la poesía, así lo expresa el siguiente parlamento.

Félix - ¡La poesía no muere! ¡No! ¡Ella vence a la muerte, al tiempo y a la vida!... ¡Se disolverá en el aire un día el sórdido gemido, de mi carne, y quedarán de mí, eternos, el júbilo, la música ideal que tiene alma!... iY es mío el alba de oro!

En relación con la construcción dramática, las acciones externas conducen al desenlace y culminación de las acciones internas que están llenas de mucho dramatismo y emoción. El segundo conduce hacia la anagnórisis con relativa calma y aceptación de los hechos y el tercero, tiene un giro dramático que se precipita hacia un desenlace inesperado en el final del tercer acto.

Félix - ¡Y mi desolación está siempre delante de mí!... ¡Tantos sueños y vidas mutiladas a mi alrededor!... Mi madre muerta en abandono... Stella, sepultada en soledad... Emelina, hundida en el vacío. Francisca, perdida en lo oscuro de su propia tristeza. Hijos enterrados en el campo... iToda mi existencia abrazada por la angustia!...

La angustia existencial hace que el ser se sienta limitado y recurra a todas las posibilidades para alcanzar mediante el amor y la fe, el sosiego, la quietud y tranquilidad que necesita ante ese momento crucial como lo es la muerte.

\section{C- El protagonista como portador de la acción}

El personaje Félix (el protagonista) es el portador de la acción desde su presentación, durante el proceso hasta la culminación del conflicto. 
La funcionalidad del espacio y acontecimiento en el mundo cerrado de la obra contribuyen a caracterizar y explicar el comportamiento de Félix: "...Prefiero este aislamiento, este silencio, esta soledad... pero sin médicos asesinos a mi alrededor". El carácter unitario de la obra se revela incluso en su relación con el título. Este es muy significativo, expresado en una oración valorativa (como la mayoría de las obras de Steiner) que designa al personaje principal, en este caso La agonía del "poeta" que lo hace diferente, excepcional, único, dándole un carácter mítico.

La visión del mundo plasmada en la obra La agonía del poeta, corresponde a la visión del mundo del autor que desde el punto de vista religioso presenta a un Félix temeroso hacia lo desconocido, a la angustia de vivir y a lo que hay después de la muerte. Reflexiona sobre la existencia de Dios y la existencia misma del hombre y las cosas en la tierra. Todo esto sumado a los factores históricos - biográficos del poeta Rubén Darío. Es posible observar la presencia de motivos y elementos existenciales, entre ellos: frustración, falta de amor, desconfianza, temor, odio, angustia, dolor, muerte, etc.

En el análisis del texto se muestra la realidad del poeta (Félix) su relación con su madre, Rosa Sarmiento, y sus esposas Rafaela Contreras, (Stella) y Rosario Murillo, (Emelina). Los conflictos del autor (Steiner) en su relación con su madre (María Teresa Sánchez) y su esposa (Margarita). Todos estos elementos acompañados de la creación del autor (ficción) le permite recrear los últimos momentos de Rubén Darío y sus vivencias personales para elaborar su obra. Steiner toma como símbolo la figura de Rubén Darío para representar la agonía no sólo del poeta, sino de la humanidad con todos sus escrúpulos y egoísmos que le hacen ser cruel, despiadado e hiriente, con su propia individualidad, y como todo ser humano se aferra a lo material. Por muy preparado que se encuentre para la hora final, siempre sentirá la incertidumbre y angustia hacia lo desconocido, por lo incierto, el vacío, la nada.

En relación con los otros personajes, éstos tienen rasgos de representatividad, aún cuando no son personajes tipo. Emelina, posee algunos elementos portadores de valores positivos y negativos de la sociedad que rodea a Félix. En el desarrollo de la acción, lo más significativo es el cuidado y atención hacia su esposo (Félix) y la comunicación o incomunicación que provoca en su relación, hecho que hace posible la existencia y el refugio en los recuerdos (lo onírico, el delirio) del enfermo.

Emelina, representa la mirada del otro. El rechazo de Félix y el odio - amor que la obsesiona. Hay momentos insoportables de Félix, ante la presencia del otro (Emelina). Es esa relación entre las conciencias de ambos lo que provoca el conflicto de Félix, lo que a su vez es el dominio de la situación por parte de Emelina y el triunfo por recuperar lo que sabía perdido. Emelina se muestra en algún momento, insensible al dolor de Félix. Su amor propio, la hace sentirse rechazada, aún en los últimos momentos de agonía del poeta y esto le causa una profunda soledad y vacío.

La mujer, se asocia con la madre del poeta, Rosa Sarmiento. Steiner, la presenta como un personaje que tiene sus propios conflictos internos. Ella tuvo que enfrentar a la sociedad de su época, aceptar una relación matrimonial que no deseaba y una maternidad inesperada. "El conflicto de Rosa, su inconformidad y su rebeldía, tiene su origen en la falta de amor en la pareja" (Palacios, 1998:174). Es un personaje triste, ansioso de ayudar al hijo en su sufrimiento, quiere dar consuelo y conducirlo hacia su último viaje. 
Stella, fue su primera esposa, muerta prematuramente en 1892, esto le causó profunda amargura al poeta. Stella conducirá a Félix, por el mundo sobrenatural de los cielos. En Rubén Darío y su creación poética Arturo Marasso, (1973) anota:

En los raros, antes de enumerar las mujeres de Poe, Darío recuerda a Stella: Alma dulce reina mía, tan presto ida para siempre... Frente al balcón vestido de rosas blancas, por donde en el paraíso asoma tu faz de generosos y profundos ojos, pasan tus hermanas y te saludan con una sonrisa, en la maravilla de tu virtud, ¡Oh mi ángel consolador, oh mi esposa! (p. 105)

Stella representa para el poeta esa armonía, esa paz y consuelo que tanto anhela.

El espacio y el tiempo de La agonía del poeta, al igual que los de la Trilogía del matrimonio, es un espacio cerrado, delimitado a cuatro paredes de una habitación (la alcoba, la oficina, la casa). La acción dramática no se caracteriza por la tensión hacia el futuro, sino que avanza lenta y realiza analepsis, retornos en el tiempo que explican el comportamiento de Félix o de los antagonistas (la mujer - madre) y las razones que les obligaron a actuar de esa manera. Postrado en el lecho de enfermo Félix, (el protagonista) se debate entre la vida y la muerte. Desde que inicia la obra hasta que termina, Félix permanece en la cama, solo con sus temores, visiones y espectros que le acechan hasta el momento final. Al culminar el II acto se da una prolepsis que muestra a Félix una visión de lo que será su cuerpo después de muerto.

Félix - iHe soñado que manos ambiciosas y sacrílegas ensuciaban las calles, enrojecían las baldosas con la sangre de mi cerebro arrancado de mi cabeza!...

Según Heidegger, de todos los entes del mundo, el Dasein es el único que sabe de su propia muerte. Morir para él no es un mero perecer, sino un modo de ser hacia la muerte. (Robert, 1973: 208). Frente a la ansiedad de la muerte surgen dos actitudes posibles: La inauténtica, que trata de ocultarnos lo que nos reserva el futuro y la auténtica que es la recuperación del ser total. En la existencia auténtica se encuentra una comprensión fundamental de la estructura temporal del Dasein. Félix experimenta estos dos momentos vitales: la permanencia, la muerte que significa el perpetuarse a través del ideal y pasión que significó su proyecto de vida, la poesía y la muerte como un hecho ineludible de angustiosa realidad, en la cual no tiene elección ni poder para decidir sobre su destino.

El conflicto de Félix al enfrentar a la muerte como una realidad ineludible de la vida concreta, es y ha sido a través de su existencia una amenaza permanente: la destrucción de lo más preciado para él, la conciencia de existir.

El pensamiento existencial no descarta la angustia ante la muerte, como experiencia reveladora de la vida y del destino personal. Considera este sentimiento de angustia necesario para devolver la autenticidad de la existencia del hombre que es la conciencia de querer regresarlo a sus más auténticas posibilidades de ser así como la capacidad de poder oír, no con el fin de regresar al pasado, sino para poder proyectarse hacia delante, al futuro a pesar del estado de angustia en que se encuentra el ser. En el reconocimiento de sus actos Félix encuentra la posibilidad de la muerte, llevado por las circunstancias a situaciones límites de odio, rencor, glorias e insatisfacciones, que le amenazan y ahogan cuando realmente su destino es arrastrarlo fatalmente hacia la muerte, y así lo expresa:

Félix -... ;Oh no quiero pensar ni imaginar nada!

...Pero, pensar y sentir es el castigo del hombre... ¿Cómo evitarlo?... y que extrañas son las formas que cobra, a veces nuestra conciencia. 


\section{CONCLUSIONES}

En la interpretación del contenido de la obra La agonía del poeta de Rolando Steiner, observamos que el autor no plantea situaciones preconcebidas, que la vida del ser transcurre en una confrontación constante y que el hombre inmerso en las diversas situaciones está apoyado en un mundo sin salida.

Sus personajes viven en un espacio cerrado, asfixiante donde la única salida para liberar la angustia y la soledad es la muerte. Los conflictos internos de sus personajes no solo son los que experimentan en el presente, sino que el recuerdo de las acciones pasadas, causa más dolor, soledad y angustia. La preocupación por el ser auténtico lleva al personaje a un análisis de la conciencia y la autocrítica constante. Se busca a sí mismo en soledad, y desde el fondo de su ser pretende lograr un sentido mas auténtico de solidaridad. El conflicto de Félix al enfrentar a la muerte como una realidad ineludible de la vida concreta es, y ha sido durante toda su existencia, una amenaza permanente. La destrucción de lo más preciado para él, la conciencia de existir.

La agonía del poeta, es la puesta en escena de los últimos instantes del protagonista Félix Rubén, la auto revisión o como decía el propio Darío en uno de los nocturnos... "ser la auto pieza / de disección espiritual, el auto - ¡Hamlet! / Diluir mi tristeza..."

La postura de Félix ante el vacío y la muerte es auténtica, en que su estado anímico de soledad, angustia existencial y ansiedad recupera definitivamente el ser para la muerte, como una permanencia sin regreso: Pero el yacer frente a ese abismo también le da conciencia de un pasado que se debate como lejana verdad en sus labios: "... No temo a la muerte...En ocasiones he gozado como no lo han logrado millonarios de esta tierra ¡he comido y vestido como príncipe, y como ellos, me he relacionado con las personalidades del mundo... He derrochado dinero, que gané en abundancia y con frecuencia he sentido el aletazo de la gloria... ¿Qué me queda por desear?.. ¿Decrepitud? ¿ldiotez senil? ¡No! Que venga la muerte. ... ¡La muerte existe, y no es dormir!...(Steiner, 1977). 
Abbagnano, N. (1995). Filosofía de lo posible. Tr. de J. Hernández Campos. A Rossi y P. Duno. México. D.F: Fondo de cultura Económica.

Arrien, J. (1978). Filosofía del Hombre. Managua: Editorial Universidad Centro Americana.

Aranda, L. R (1974). Filosofía de la existencia. Buenos Aires: Ediciones Aguilar.

Blanchot, M. (1992). El espacio Literario. Barcelona: Ediciones Paidos,

Bettetini, G (1975) Producción Significante y Puesta en escena. Barcelona: Editorial Gili S.A.

Darío Rubén. (1992). El mundo de los sueños. Ediciones y notas de Ángel Rama. Publicado en Madrid en 1917. Ministerio de Educación.

De Toro, A (1990) Texto - Mensaje - Recipiente. Análisis Semiótico estructural de textos narrativos dramáticos y líricos. Buenos Aires: Editorial Gaterna.

Lawson, J. H (1976) Teoría y técnicas de la dramaturgia. La Habana: Editorial Arte y Literatura.

Marasso, A. (1973) Rubén Darío y su creación poética. Buenos Aires - Argentina: Editorial Kapeluz,

Mier R. (1990) Introducción al análisis de textos. México: Editorial. Trillas.

Navarrete. E. (1991, 9 de febrero) "La agonía del poeta” Prensa Literaria.

Palacios, N. (1998). Voces femeninas en la narrativa de Rosario Aguilar. Managua: Editorial Ciencias Sociales.

Pollmann, L. (1973). Sartre y Camus. Literatura de la existencia. Madrid: Editorial Gredos.

Raeymaeker de L. (1968). Filosofía del ser. Ensayo de Síntesis metafísica. Versión española de Maria Dolores Mouton y Valentín García. Madrid.

Robert, G. (1973). Temas existenciales en la novela española de posguerra. Madrid: Editorial Gredos

Sartre, J. P. (1964). Kierkegaard vivo. Madrid: Alianza Editorial

Steiner, R. (1977). La Agonía del Poeta. El pez y la serpiente.

Talens, J. (1995). Elementos para una semiótica del texto artístico. (Poesía, narrativa, teatro, cine) (5 ed.). Madrid: Ediciones Cátedra. 


\section{REFERENCIAS}

Torres, E. (1983). La dramática vida de Rubén Darío. Managua: Editorial Nueva Nicaragua.

Tamayo, M. (1993). El proceso de la investigación científica. (2a ed.). México: Editorial Limusa, S. A.

Urtecho, A. (1991, 19 de enero) “Steiner. Mascara y Carcajada”. Prensa Literaria.

Valle, P. Y Ander - Egg, E. (1997) Guía para preparar monografías y otros textos expositivos. Editorial Lumen - Hymanitas, Argentina.

Valessi, A. (1991, 21 de mayo) "Steiner y el Teatro de la Crueldad" Prensa Literaria. 